$+4 \mathrm{~mL}$ [95 CI: $-13-+19], p=0.61$ ) or median infarct growth ( $1 \mathrm{~mL}$ versus $5 \mathrm{~mL}$, estimated difference $1.0 \mathrm{~mL}$ [95 CI: -7.0 +10 ], $p=0.71$ ). For decision to treat up to 20 hours after onset, time did not correlate with final infarct volume, Figure 1. Similar rates of complications and mortality were observed.

Conclusion Using MR selection, similar good outcomes after thrombectomy for ACLVO stroke may be achieved well beyond 6 hours.

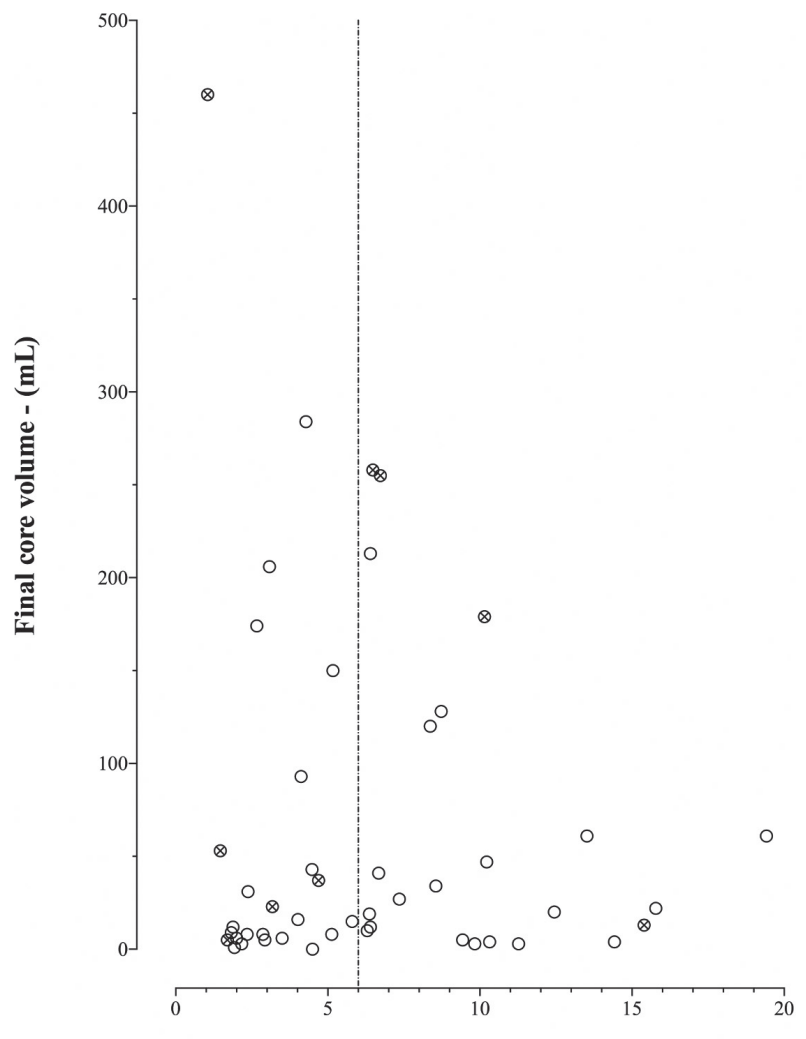

Treatmend decision time from onset - (h)

Abstract P005 Figure 1 Final core volume does not correlate with treatment decision time. Dashed line, $\mathrm{t}=6$ hours; open circle $=$ successful recanalization, closed circle $=$ failed recanalization; Spearman rank-order correlation, $r_{\mathrm{s}}(46)=0.085, p=0.566$

Disclosures B. Cristiano: None. M. Pond: None. S. Basu: None. U. Oyoyo: None. J. Jacobson: 4; C; GeneLux.

\section{P-006 PRESENTING DIFFUSION-RESTRICTED CORE VOLUME, NOT TIME, PREDICTS FINAL INFARCT VOLUME AFTER THROMBECTOMY IN ANTERIOR CIRCULATION LARGE VESSEL OCCLUSION STROKE}

B Cristiano, M Pond, S Basu, U Oyoyo, J Jacobson. Neuroradiology, Loma Linda University Hospital, Loma Linda, CA

10.1136/neurintsurg-2016-012589.48
Purpose With thrombectomy for anterior circulation large vessel occlusion (ACLVO) stroke, time to recanalization is considered important, but collateral status may be a more important driver of outcome than time. Here we used data from an institutional cohort to test the hypothesis that small diffusionrestricted core volume on presenting MRI, a biomarker of robust collateral perfusion, is the dominant predictor of favorable outcome after thrombectomy.

Methods A cohort of 56 ACLVO patients treated with thrombectomy at our institution between $11 / 1 / 2012$ and 5/15/2015 was studied. Seven early-presenting patients with contraindication to MRI proceeded immediately to thrombectomy upon CTA confirmation of LVO. Forty-nine patients with CTA-proven LVO but no MR contraindication went next to MRI and were selected for thrombectomy based on low diffusionrestricted infarct volume (non-intervention threshold: core volume $>$ (100 minus patient age) $\mathrm{mL}$ ). Final infarct volume was measured on post-treatment imaging. Groupwise comparisons were made between patient subgroups. A logistic regression was performed using final infarct volume $\leq 50 \mathrm{~mL}$, a validated predictor of good clinical outcome, as the outcome variable.

Results For decision to treat up to 20 hours after onset, time did not correlate with final infarct volume (Spearman rankorder correlation, $\left.r_{s}(46)=0.085, p=0.566\right)$. Despite significantly earlier treatment decision times $(3.0 \mathrm{~h}$ [2.5-3.6] vs. 6.4 $\mathrm{h}$ [2.9-8.7]), median final infarct volume was larger in the early CTA-only screened patients compared with MR-selected patients $(134 \mathrm{~mL}$ vs. $20 \mathrm{~mL}$, estimated median final infarct difference of $100 \mathrm{~mL}$ [95 CI: 1.00-134], $p=0.043)$. The odds of a final core infarct greater than $70 \mathrm{~mL}$, a poor prognostic factor, were significantly lower in the MR-screened group compared with a population of early patients screened with CTA only OR 0.013 ([95 CI: 0.022-0.757], $p=0.022$ ). In a univariate logistical regression, only presenting core volume, presenting core volume $\leq 50 \mathrm{~mL}$, age, and post procedure TICI $\geq 2 \mathrm{~B}$ were significant predictors of a small completed infarct. In a multiple logistic regression model, presenting core volume $\leq 50 \mathrm{~mL}$, not time to recanalization, was the dominant predictor of small final infarct volume (OR 15.2 [95CI $1.3,180]$ vs. 0.94 [95CI $0.78,1.1]$ ). A 3 variable logistic regression model incorporating presentation core volume, age, and TICI $\geq 2 \mathrm{~B}$ as covariates was statistically significant $\chi^{2}(2)=22.65,(p<0.001)$ and accounted for $52.9 \%$ of the variance in outcome with a percent accuracy of classification of $85.4 \%$.

Conclusion Applying multiple analytic methodologies, we are unable to detect any significant interaction between time from symptom onset and final infarct volume in this institutional cohort of 56 ACLVO patients treated with thrombectomy, most of whom were selected for treatment based on MR screening with DWI. Importantly, favorable final infarct volumes were observed among MR selected patients treated up to 20 hours from symptom onset. Well beyond commonly accepted thresholds. Consideration should be given to patient selection strategies incorporating MRI with diffusion weighted sequences rather than time from symptom onset alone.

Disclosures B. Cristiano: None. M. Pond: None. S. Basu: None. U. Oyoyo: None. J. Jacobson: 4; C; GeneLux. 